\title{
Visual Perception Model for Sense of Materials
}

\author{
Wenhao Wang and Toshikazu Kato \\ Faculty of Science and Engineering, Chuo University \\ 1-13-27 Kasuga, Benkyo-ku, Tokyo, 112-8551 Japan \\ wang_wenhao@indsys.chuo-u.ac.jp, kato@indsys.chuo-u.ac.jp
}

\section{Introduction}

Natural surfaces has a different materials, human can instantly feel these characteristics. For example, display of fruit on the supermarket shelves, we can determine only the eyes whether they are fresh. When shopping, we can quickly determine the quality of material goods, as well as light and heavy, soft and hard, and so on. We call this feeling "SITUKAN", it is Japanese and means sense of materials.

In a few years ago, NTT Communications what the Japanese companies did research on sense of materials. They published the results in the Nature [1], and said the brightness is an important reason of sense of materials. They used global features depicts the glossiness and semi-transparent of success. However, after our research found that, classify the glossiness and semi-transparent, local features more efficient than global features.

\section{Experiment}

\subsection{Methods}

\section{Local and Global Features}

Brightness histograms are widely used as global graphical features which show the distribution of brightness in image plane. Nevertheless, in visual perception process, photoreceptors extract not only global features, but local features such as brightness, luminance, edges and contrasts. By integrating and selecting global and local features extracted by photoreceptors, human beings perceive textures and shapes. Therefore, we should design local features to describe relations in neighboring pixels as well as global features.

Although we can measure the relations of reference pixel $\gamma$ on image plane $\mathrm{P}$ and $\mathrm{N}$ pixels around $r\left(\mathrm{a}^{1}, \ldots, \mathrm{a}^{\mathrm{N}} \in \mathrm{R}^{2}\right)$, we think relations in neighboring pixels properly show local features. Therefore, we adopted $3 \times 3$ pixel window to compute local features of directions and curvatures of differentials. Fig. 1 shows the 28 local mask patters: "+" represents the reference pixel $r$ and "*"represents displacement vectors $a_{1}^{(i)}, a_{2}^{(i)} \ni R^{2}(i=1, \ldots, 28)$.

Even if capturing the same texture, the camera angle and the lightning conditions are not same. In addition, images in database are not always noiseless. If graphical features are sensitive to camera angle and noises on images, we can not apply them to 


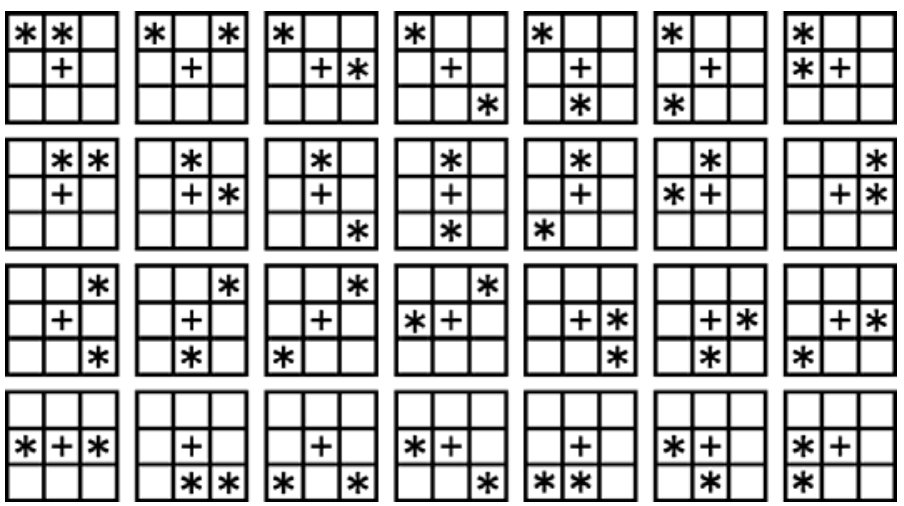

Fig. 1. Local mask patterns for graphical features

similarity image retrieval. Therefore, we should design shift-invariant and noiserobust graphical features. When photoreceptors receive stimulus, they send a signal to higher level of visual perception process. A receptor receiving stimulus also sends signals to neighboring photoreceptors, telling them to turn down their own sensitivity to stimulus. This antagonistic interaction between a photoreceptor and its surround is often called lateral inhibition. Lateral inhibition is a mechanism which enhances contrast between stimuli.

We defined tri-contrast as a mathematical model of lateral inhibition function by

$$
\begin{aligned}
& \operatorname{Cont}^{(i)}\left(\mathbf{a}_{1}^{(i)}, \mathbf{a}_{2}^{(i)}, \mathbf{r}\right) \\
& =\frac{\left\{f\left(\mathbf{r}+\mathbf{a}_{1}^{(i)}\right)-f(\mathbf{r})\right\}+\left\{f\left(\mathbf{r}+\mathbf{a}_{2}^{(i)}\right)-f(\mathbf{r})\right\}}{\left|f\left(\mathbf{r}+\mathbf{a}_{1}^{(i)}\right)\right|+\left|f\left(\mathbf{r}+\mathbf{a}_{2}^{(i)}\right)\right|+2|f(\mathbf{r})|}(i=1, \ldots, 28)
\end{aligned}
$$

where $\mathrm{a}_{1}$ and $\mathrm{a}_{2} \in \mathrm{R}^{2}$ are the displacement vectors (each "**" in Fig. 1 denotes $\mathrm{a}_{1}$ and $\mathrm{a}_{2}$ ), function $f(\mathrm{r})$ is stimulus defined on independent three-dimensional color space, and $\mathrm{r} \in \mathrm{P}$ is the image coordinate vector (each"+" in Fig. 1 denotes $\mathrm{r}$ ). A denominator and a numerator of Eq. (1) denote stimulus intensity and difference of stimulus, respectively. Since stimulus intensity will become stronger if stimuli of pixels around reference pixel $f\left(\mathrm{r}+\mathrm{a}_{1}^{(\mathrm{i})}\right), f\left(\mathrm{r}+\mathrm{a}_{2}^{(\mathrm{i})}\right)$ become strong, photoreceptor's response $\operatorname{Cont}^{(\mathrm{i})}\left(\mathrm{a}_{1}^{(\mathrm{i})}, \mathrm{a}_{2}^{(\mathrm{i})}, \mathrm{r}\right)$ will become weaker. In addition, since tri-contrast value is normalized by stimulus intensity, it is scale-invariant to stimulus intensity and robust to noise.

Lateral inhibition exists not only in photoreceptors but in various ganglion of higher level of visual perception process. Therefore, we think that lateral inhibition is useful to select and emphasis dominant factors extracted by photoreceptors.

\section{Grayscale Photo}

In our experiment, use the same size and shape of objects as the subject. One is painted with a gloss coating over the object, and the other is not treated semitranslucent objects. We were on the two objects can turn the disc. Whenever the rotary disk 2 degrees to take a photo with grayscale mode camera (Fig. 2). Then, we 
shot a total of 360 photos were divided into 2 groups. Group A is glossiness and Group B is semi-transparent sense. Finally, we will remove all the photos in the background, because this background has nothing to do with the sense of materials.
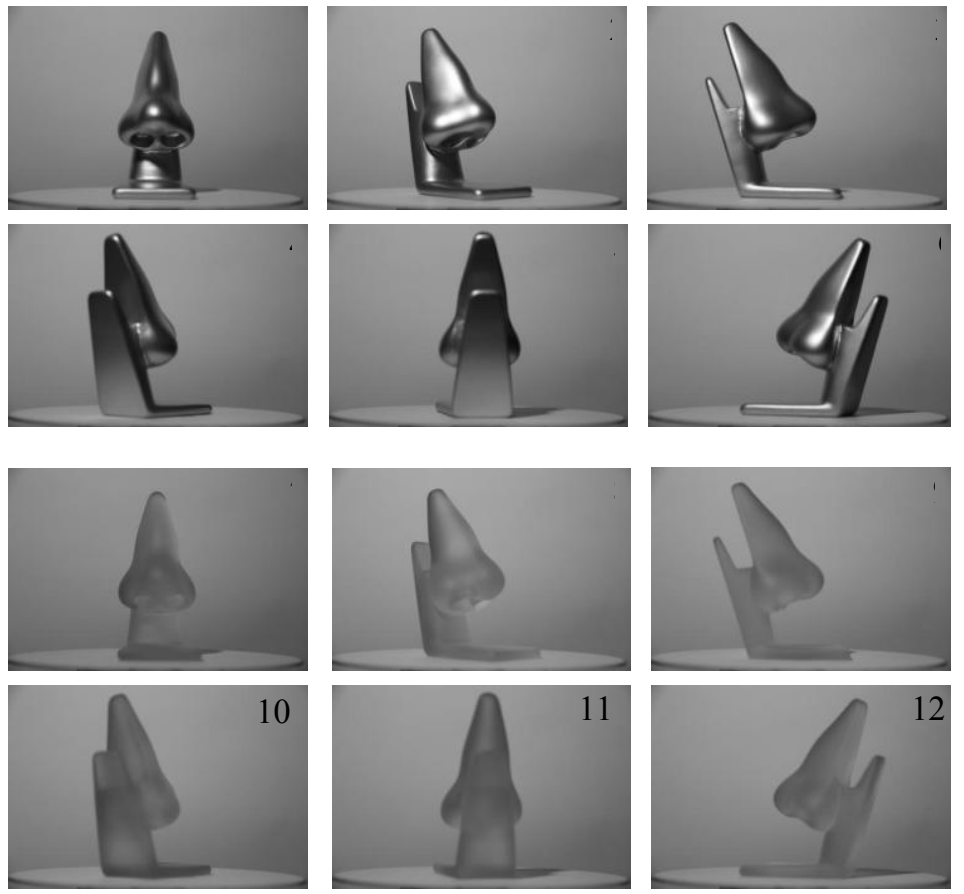

Fig. 2. 1-6 photos is part of Group A, 7-12 is part of Group B

\section{Procedure}

First, we took out all the photos from the local and global features (Fig. 3), and put the data normalization. Next, we arbitrarily removed from each group (Group A,B) in
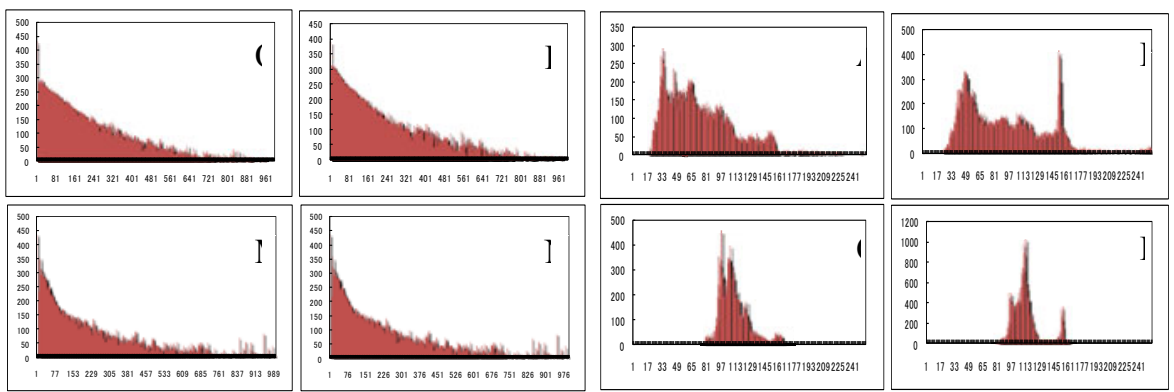

Fig. 3. $\operatorname{Local}(\mathrm{O}, \mathrm{P}, \mathrm{M}, \mathrm{N})$ and $\mathrm{Global}(\mathrm{A}, \mathrm{B}, \mathrm{C}, \mathrm{D})$ features of brightness histograms 
30, 60, 120 photos and put them into 3 samples as teaching data. Finally, let the computer after learning of the two features to classify all photos.

\section{Results}

Table. 1 shows that, with the reduction in the amount of teaching data, global features gradual decline in accuracy, but the correct rate of local features is not much change. Especially in the teaching data for the 30, the difference between the correct rate of $20 \%$. And both are used, the correct rate has remained at $100 \%$. This data clearly shows that classify the glossiness and semi-transparent, local features more efficient than global features.

Table 1. Teaching data classification accuracy

\begin{tabular}{|l|c|c|c|}
\hline & 120 photos & 60 photos & 30 photos \\
\hline 1. Local features & $98 \%$ & $99 \%$ & $98 \%$ \\
\hline 2. Global features & $89 \%$ & $80 \%$ & $78 \%$ \\
\hline $1+2$ & $100 \%$ & $100 \%$ & $100 \%$ \\
\hline
\end{tabular}

\section{References}

[1] Motoyoshi, I., Nishida, S., Sharan, L., Aselson, E.H.: Image statistics and the perception of surface qualities. Nature 447 (2007)

[2] Oguma, M., Tsuboi, M., Horikoshi, T., Takaki, Y.: Subjective Evaluation of Appearance Reproduction of Moving 3D Images Produced by High-Density Directional Display. ITE 33(16), 1-4 (2009)

[3] Spillmann, L., Werner, J.S.: Visual Perception. Academic Press, London (1990) 